\title{
Modificação de celulose e avaliação da sua ação no fenômeno de parafinação em petróleo
}

\author{
Cellulose modification and evaluation of its action in the waxing phenomenon in \\ petroleum \\ C. M. R. Santos*; D.C.S. da Graça; G. Cardoso \\ Departamento de Engenharia Química/Laboratório de Desenvolvimento e Caracterização de Materiais/ Universidade \\ Federal de Sergipe,CEP,49100-000 São Cristóvão-SE, Brasil \\ *e-mail: cleciosantos-1@hotmail.come giselia@ufsbr \\ (Recebido em 21 de março de 2016; aceito em 22 de abril de 2016)
}

\begin{abstract}
A celulose é uma substância natural, macromolecular, não tóxica, de origem renovável e biodegradável, cuja estrutura química pode sofrer modificações através da substituição das hidroxilas presentes na sua macromolécula e assim, obter produtos com variadas propriedades de uso. Com o aumento da atenção mundial para a degradação ambiental, a obtenção de novos produtos a partir de modificação química da celulose tem sido realizada com crescente aplicabilidade em diversos setores industriais, dentre eles, a indústria de extração e produção de petróleo. Este estudo mostra a ação de substância surfactante obtida a partir de xantato celulósico de sisal (Agave sisalana) modificado com álcool láurico no fenômeno de parafinação de petróleo pesado, em bancada de laboratório, por meio da medida de temperatura de iniciação de aparecimento de cristais de parafina (TIAC) e da energia de ativação de fluxo. Os resultados dos referidos experimentos mostraram que este não reduziu os valores de TIAC em amostra de óleo pesado em estudo, mas promoveu redução de até $8 \mathrm{~kJ} / \mathrm{mol}$ na energia de ativação de fluxo para o petróleo parafínico aditivado com 1000ppm de celulose modificada na presença da salmoura.
\end{abstract}

Palavras chave: Celulose; aditivo; parafinação.

Cellulose is a natural substance, macromolecular, non-toxic, renewable and biodegradable origin, whose chemical structure can be modified by replacing the hydroxyls present on the macromolecule and thus obtain products with varying properties of use. With increasing worldwide attention to environmental pollution, obtaining new products from chemical cellulose modification has been carried out with increasing applicability in various industries among them the extraction and production of oil industry. This study shows the action of a surfactant substance obtained from sisal cellulose xanthates (Agave sisalana) modified with lauric alcohol, in laboratory bench, the heavy oil waxing phenomenon through the appearance initiation temperature measurement paraffin crystals (TIAC) and flow activation energy. The results of these experiments showed that this did not reduce the TIAC values in heavy oil sample in question but promoted reduction of up to $8 \mathrm{~kJ} / \mathrm{mol}$ in the flow activation energy for paraffin oil additivated with modified cellulose in the presence of brine 1000ppm.

Keywords: cellulose; additive; waxing

\section{INTRODUÇÃO}

A injeção de fluidos contendo aditivos para correção de perfil de escoamento de óleos pesados representa uma das diversas técnicas que permitem alcançar, com objetividade, a melhoria das condições de escoamento de hidrocarbonetos, na indústria de petróleo, em todos os seus processos, desde a rocha reservatório até o refino [1-3].

As propriedades que devem caracterizar o fluido contendo aditivo na produção de petróleo estão diretamente ligadas ao poder de surfactar e emulsificar o óleo, dando-lhe equilíbrio hidrodinâmico para adquirir mobilidade e ser arrastado da rocha reservatório para o poço e deste até o tanque de armazenamento, além de melhorar a interface água/petróleo e vice-versa. Um aditivo para atender essas necessidades ao longo do escoamento de petróleo pesado deve 
apresentar características de não inflamabilidade na faixa de temperatura de trabalho, possuir $\mathrm{pH}$ básico, ser não cáustico e hidrossolubilidade [4,5].

O início do fenômeno de precipitação de parafinas, em óleo pesado, ocorre pela aproximação entre cadeias longas de hidrocarbonetos cujas interações se dão por forças intermoleculares que por consequência da diminuição da temperatura, do fundo até a superfície do poço, favorece a formação de núcleo de parafina e posteriormente precipitação na forma de cera. Contudo, a aplicação de aditivos químicos durante a produção de petróleo influencia nas propriedades das interfaces de sistemas multifásicos presentes de forma definitiva. Os aditivos químicos comumente empregados como perturbadores de crescimento de cristais de parafinas, não só surfactantes sintéticos, mas também substâncias como biossurfactantes, lípidos e macromoléculas, são substâncias ativas em interface de sistemas multifásicos [6-9].

Para aplicar substâncias surfactantes em processos tecnológicos, uma caracterização quantitativa do seu comportamento interfacial é necessária. Isto inclui caracterizar propriedades do sistema no estado de equilíbrio, bem como as propriedades no estado de não equilíbrio. Na maioria dos casos, a aplicação de surfactantes ocorre em condiçãode estado de não estacionária e por isso as propriedades dinâmicas possuem maior relevância para o controle do processo. Um grande número de estudos tem sido realizadopara avaliar o comportamento dinâmico do escoamento de petróleo através de medida de propriedades térmicas,parâmetros reológicos, identificação da estrutura morfológica do precipitado petrolífero e da concentração crítica do aditivo químico empregado [10].

Nos últimos anos, como forma de obter aditivos químicos com menor toxidade e maior biodegradabilidade, vem sendo estudado a aplicação de líquidos iônicos, como solvente alternativo em meio reacional para uma grande variedade de processos de modificação e transformações em celulose [11-13].

A reatividade da celulose é governada por sua estrutura química e física que apresenta grupos hidroxilas que podem reagir com reagentes de adição, substituição e oxidação; grupos acetais que podem sofrer hidrólise tanto em meio ácido como em meio alcalino e grupos aldeídicos terminais que podem ser reduzidos para grupos alcoóis, oxidados para grupos carboxilas, ou, quando na presença de álcali, rearranjados para formar grupos finais alcoólicos ou carboxílicos [14-17].

As reações químicas mais importantes da celulose são as reações de ligações glucosídicas (degradação da celulose); reações de adição e reações de substituição. As reações de ligações glucosídicas ocorrem com o rompimento das mesmas entre os monômeros de glucose. Esse rompimento, dependendo das condições físicas do processo reativo, pode se estender por toda a cadeia, o que justifica essa reação ser muitas vezes denominada de degradação da celulose. Em geral, os rompimentos das ligações glucosídicas levam ao rompimento de moléculas com menor grau de polimerização, o que afeta diversas propriedades da cadeia molecular da celulose [17].

Em geral, as reações de adição com a celulose ocorrem por meio dos agentes de intumescimento, que são compostos químicos, cuja concentração a ser adicionada depende do tipo de reagente, da temperatura e da estrutura física da celulose. Tais reações de adição se iniciam pela quebra das pontes de hidrogênio, entre as cadeias adjacentes de celulose, no decorrer do fenômeno de intumescimento. Nesta estrutura intumescida, um reagente químico pode penetrar e se propagar livremente, chegando a formar derivados de celulose relativamente homogêneos [18-19].

A modificação da celulose, para uso como aditivo corretor de escoamento de óleo pesado deve atribuir à sua estrutura química características surfactantes, isto é, estrutura molecular com caráter anfifílico, constituída de segmento hidrofóbico (apolar) e segmento hidrofílico (polar) na mesma molécula possibilitando a interação entre fases de sistemas líquidos multifásicos, favorecendo assim o seu escoamento. Daí a reação de substituição ser empregada [19].

Algumas substâncias surfactantes apresentam como porção apolar uma cadeia hidrocarbonada enquanto a porção polar pode ser iônica (aniônica ou catiônica), não iônica ou anfotérica [20]. Contudo, a formação de espumas, características que dependem dos grupos polares e apolares presentes na estrutura do surfactante, são indesejáveis no processo de produção de petróleo. Além do mais, para que os efeitos da tensão superficial sejam significativos, deve-se utilizar a substância com atividade surfactante em concentrações abaixo da sua concentração micelar 
crítica, uma vez que ao utilizar concentrações acima desta, a formação de micelas impede a atuação do surfactante na tensão superficial e esse perde a sua atividade [21-23].

Vários são os grupos de pesquisa em todo mundo que nas últimas décadas têm se interessado pela modificação de celulose, o que reflete na grande quantidade de trabalhos encontrados na literatura que relatam versáteis transformações químicas da celulose [24-26].

O presente estudo trata da obtenção de substâncias surfactantes a partir da modificação da celulose de sisal (Agave sisalana) a partir de xantatos celulósicos, posterior modificação desse com o álcool láurico, e avaliação em bancada de laboratório das suas ações na inibição de precipitação de parafina em óleo pesado na presença de salmoura sintética com composição e concentração de sais do cenário do pré-sal.

\section{MATERIAL E MÉTODOS}

\subsection{Materiais}

Os materiais utilizados nesse trabalho foram: celulose de sisal(Agave sisalana) doada pela fábrica de papel SUZANO,os reagentes comerciais da VETEC: álcool láurico, usado como modificante;ácido sulfúrico, como agente de sulfonação; hidróxido de sódio, usado na deshidroxilação da celulose a ser modificada; dissulfeto de carbono, usado como finalizador de deshidroxilação da celulose; éter etílico, usado como solvente do álcool láurico no processo de sulfonação, e resina de troca iônica da marca DOWEX MARATHON, doada pela Fábrica de Fertilizantes Nitrogenados - FAFEN/SE, cedida pelo laboratório de química industrial/DEQ-UFS, usada na remoção do íon sulfato e petróleo parafínico gentilmente doado pela Petrobras/UOSEAL - material teste da ação do aditivo de celulose modificada.

\subsection{Métodos}

\section{Mecanismo de modificação da celulose}

A modificação da celulose de sisal (Agave sisalana) se deu a partir da obtenção do xantato de celulose pelo processo proposto por Hirano[27]. Após a modificação em meio aquoso, ocorre liberação o sal sulfato de sódio $\left(\mathrm{Na}_{2} \mathrm{SO}_{4}\right)$ conforme demonstrado ilustrado na Figura 1.

Faz-se necessário a remoção dos íons sulfato na finalização do processo de modificação da celulose, uma vez que a presença destes íons em soluções aquosas, durante a extração e produção de petróleo, pode levar a formação de sais insolúveis que se precipitam e se depositam nas paredes da tubulação aumentando a energia de ativação de fluxo, e diminuindo a capacidade do seu escoamento.

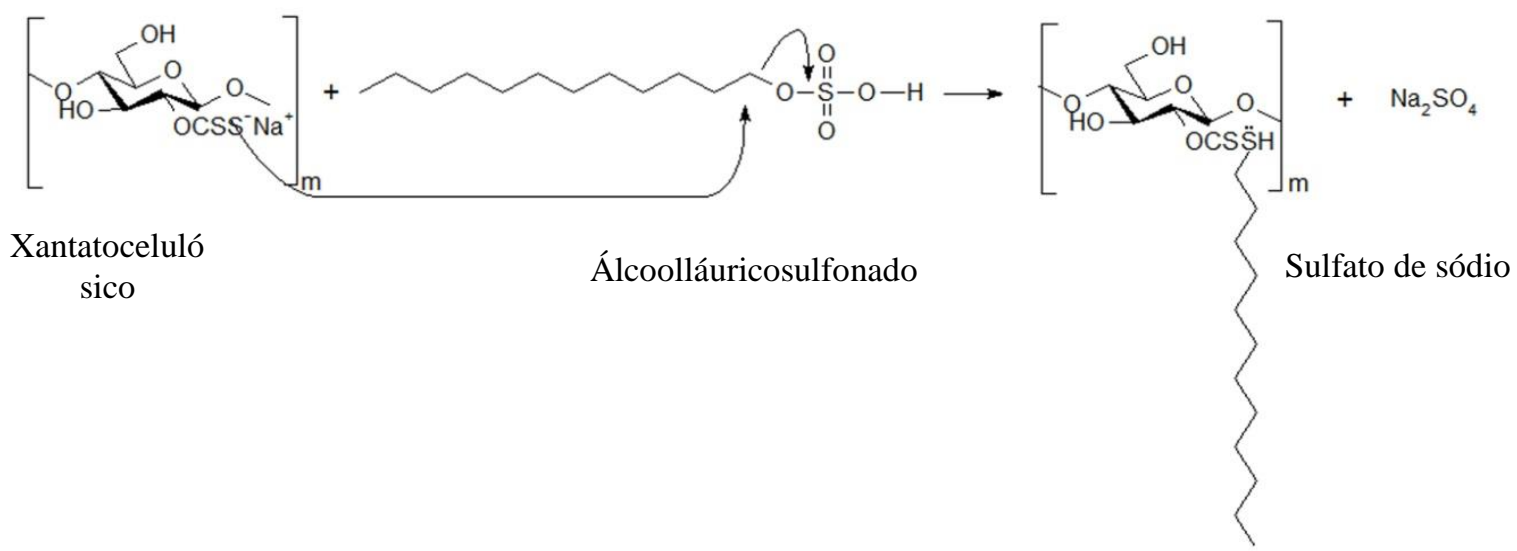

Éter de celulose

Figura 1: Representação esquemática do mecanismo proposto para a modificação da celulose a partir de xantato de celulose de sisal com álcool láurico. 


\section{Mecanismo de remoção de íon sulfato}

A remoção do íon sulfato do meio reacional presente na forma de sulfato de sódio, no final do processo de modificação da celulose, foi realizada por meio da adição da resina de troca iônica DOWEX MARATHON que devido à sua capacidade de troca iônica, adsorve o íon sulfato e se precipita no meio reacional. A equação eq.(1) mostra, de forma esquemática, a reação de remoção do íon sulfato.

$$
\mathrm{Na}_{2} \mathrm{SO}_{4}(\mathrm{aq})+2 \mathrm{ROH}(\mathrm{s}) \rightarrow 2 \mathrm{RSO}_{4}^{2-}(\mathrm{aq})+2 \mathrm{NaOH}(\mathrm{aq})
$$

A eficiência do processo de remoção do íon sulfato foi verificada por meio da identificação da presença do íon sulfato por titulometria, empregando como titulante, uma solução de cloreto de bário $10 \%(\mathrm{~m} / \mathrm{v})$ para o analito íon sulfato presente em amostra de solução aquosa $1 \%(\mathrm{v} / \mathrm{v})$ de celulose modificada, antes e após o processo de tratamento de remoção do íon sulfato por resina de troca iônica. A comprovação da presença do íon sulfato ocorreu pela constatação de formação do sulfato de bário por meio de observação de turvação da solução titulada.

A eq.(2) mostra de forma simplificada a possível formação de sulfato de bário no sistema em estudo.

$$
\mathrm{BaCl}_{2}(\mathrm{aq})+\mathrm{NaSO}_{4}(\mathrm{aq}) \rightarrow \mathrm{BaSO}_{4}(\mathrm{~s})+2 \mathrm{NaCl}(\mathrm{aq})
$$

\section{Separação da celulose modificada do meio reacional}

A separação da celulose modificada do meio reacional ocorreu por processo de liofilização que consistiu em primeiro, congelar todo o material do meio reacional, em seguida submeter a fase aquosa sólida à sublimação sob pressão reduzida, finalizando o processo com a remoção da fase aquosa incongelável (adsovida na celulose modificada) por dessorção, através de secagem à vácuo.Obtendo-se assim como produto final a celulose modificada, na forma de pó [28].

Para a realização do congelamento da amostra utilizou-se o ultrafreezer da marca Liotop, modelo UFR 30. A sublimação e a dessorção, respectivamente, da fase aquosa congelada e adsorvida na celulose modificada, se deu por meio do equipamento liofilizador da marca Liotop, modelo L101, à pressão reduzida de $250-500 \mu \mathrm{mHg}$, na temperatura de $-50^{\circ} \mathrm{C}$.

\section{Caracterização química da celulose modificada}

Análise espectroscópica de absorção na região do infravermelho com Transformada de Fourier (FT-IR) foi realizada para identificação da inserção do agente modificante à cadeia da celulose, utilizando o equipamento da marca Thermo Scientific modelo Nicoletti S10, no modo ATR.

\section{Determinação da concentração micelar crítica da celulose modificada}

A condutividade em uma solução é a medida da mobilidade das espécies iônicas e depende do número de íons presente. Para eletrólitos fortes, a concentração de íons na solução é diretamente proporcional à concentração de eletrólito adicionado à solução, enquanto que para eletrólitos fracos, a concentração de íons na solução depende de seu equilíbrio de dissociação. A condutividade eletroquímica molar específica $\left(\mu \mathrm{S} . \mathrm{cm}^{-2} \cdot \mathrm{mol}^{-1}\right)$ medida na solução de um eletrólito fraco é, na verdade, a condutividade resultante da concentração total de eletrólito adicionado mais a contribuição do agregado micelar [29].

A determinação da concentração micelar crítica foi realizada a partir da medida de condutividade eletroquímica molar, em meio aquoso, à temperatura ambiente, da celulose modificada em diferentes concentrações.

As medidas da condutividade eletroquímica molar específica em função da concentração de surfactante foram realizadas adicionando-se pequenas quantidades de celulose modificada a um volume conhecido de água deionizada, medindo-se cada valor de condutividade de equilíbrio, por 
meio do equipamento Marconi - CA150, com solução padrão de referência de $\mathrm{KCl}$ previamente termostatizada a $25,0 \pm 0,1^{\circ} \mathrm{C}$.

Avaliação comportamento da temperatura de iniciação da cristalização (TIAC) e da energia de ativação de fluxo $\left(E_{a}\right)$ por reologia

A ação da celulose modificada no fenômeno de parafinação foi realizada por determinação da temperatura de início do aparecimento de cristais de parafina (TIAC) por medida reológica da amostra de petróleo aditivado com celulose modificada, em concentrações abaixo da concentração micelar crítica, na temperatura ambiente e de poço $\left(60^{\circ} \mathrm{C}\right)$.

Para a determinação da TIAC foram preparadas emulsões água/óleo e salmoura/óleo na proporção 20/80 e, adicionado celulose modificada nas concentrações de 250, 500, 750 e 1000 ppm. E, submetidas a ensaio de viscosidade em função da temperatura, em regime de resfriamento de $60^{\circ} \mathrm{C}$ a $25^{\circ} \mathrm{C}$, utilizando um viscosímetro da marca Brookfield, modelo $D V$ - III ULTRA. Para fins de comparação, também foram submetidas às mesmas analises, sem a adição de celulose modificada, as emulsões de água/óleo e salmoura/óleo.

A energia de ativação de fluxo foi medida, em todas as amostras em estudo, através da equação de Arrhenius, eq.(3).

$$
\mu=\mu_{\infty} \exp \left(\frac{E a}{R T}\right)
$$

Onde:

$\mu(\mathrm{Pa} . \mathrm{s})$ : viscosidade;

$\mu_{\infty}(\mathrm{Pa} . \mathrm{s})$ : viscosidade quando a temperatura tende ao infinito;

$\mathrm{E}_{\mathrm{a}}(\mathrm{kJ} / \mathrm{mol})$ : energia de ativação de fluxo viscoso;

$\mathrm{R}(\mathrm{kJ} / \mathrm{mol})$ : constante universal dos gases;

$\mathrm{T}(\mathrm{K})$ : temperatura absoluta.

A representação gráfica da eq.(3) do $\ln \mu$ versus $1 / T$ fornece uma reta, na qual o coeficiente angular é numericamente a razão $\left(\mathrm{E}_{\mathrm{a}} / \mathrm{R}\right)$ e o coeficiente linear o valor de $\ln \mu_{\infty}$. O que facilmente possibilita determinar a energia de ativação de fluxo $\left(\mathrm{E}_{\mathrm{a}}\right)$ e a viscosidade quando a temperatura tende ao infinito $\left(\mu_{\infty}\right)$. O conhecimento destes parâmetros permite verificar os efeitos da temperatura, da natureza química da celulose modificada e da concentração de sal na viscosidade e na energia de ativação de fluxo do petróleo. Maior energia de ativação de fluxo indica uma mudança mais rápida da viscosidade com a temperatura [30].

\section{Preparação da salmoura sintética}

A preparação da salmoura se deu com base nos dados de trabalhos divulgados de estudos de poços em teste de longa duração (TLD) no cenário pré-sal [31].

A Tabela 1 apresenta as concentrações dos sais utilizados na preparação da salmoura.

Tabela 1: Composição da salmoura sintética

\begin{tabular}{cc}
\hline Componentes & Concentrações $(\mathrm{g} / \mathrm{L})$ \\
\hline $\mathrm{NaCl}$ & 168,6 \\
$\mathrm{CaCl}_{2} \cdot 2 \mathrm{H}_{2} \mathrm{O}$ & 64,09 \\
$\mathrm{MgCl}_{2} \cdot 6 \mathrm{H}_{2} \mathrm{O}$ & 19,63 \\
$\mathrm{KCl}$ & 0,78 \\
$\mathrm{NaHCO}_{3}$ & 0,35 \\
$\mathrm{Na}_{2} \mathrm{SO}_{4}$ & 0,15 \\
\hline
\end{tabular}




\section{RESULTADOS E DISCUSSÃO}

As Figuras 2 (a) e (b) mostram respectivamente a celulose de sisal antes da modificação e modificada após sofrer liofilização. Observa-se que a celulose modificada se apresenta com características físicas diferentes da celulose bruta, isto é, na forma de pó homogêneo, de cor branca.

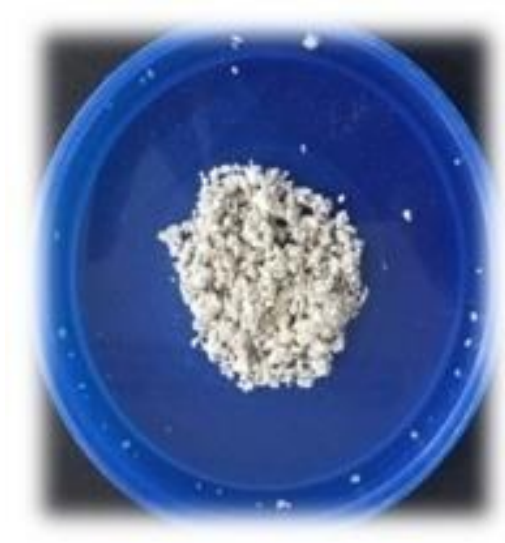

(a)

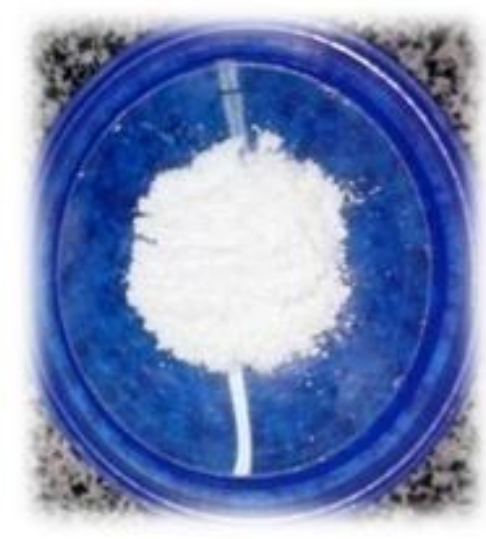

(b)

Figura 2: Amostra da celulose de sisal: (a) antes do processo de modificação; (b) modificada após o processo de liofilização.

Espectroscopia por FT-IR da celulose de sisal modificada com álcool láurico

A Figura 3 mostra os resultados da espectroscopia na região do infravermelho (FT-ir) para a celulose bruta, modificada e o agente modificante. Na análise comparativa dos espectros observase um deslocamento da banda de número de onda $1215 \mathrm{~cm}^{-1}$ característico da formação de tiocarbonila $(\mathrm{C}=\mathrm{S})$. A banda que aparece em torno de $2918 \mathrm{~cm}^{-1}$, está relacionada às ligações $\mathrm{C}$ $\mathrm{H}$, presentes em grande quantidade no álcool láurico utilizado como modificante e que aparece na celulose de sisal após sofrer modificação [32]. 


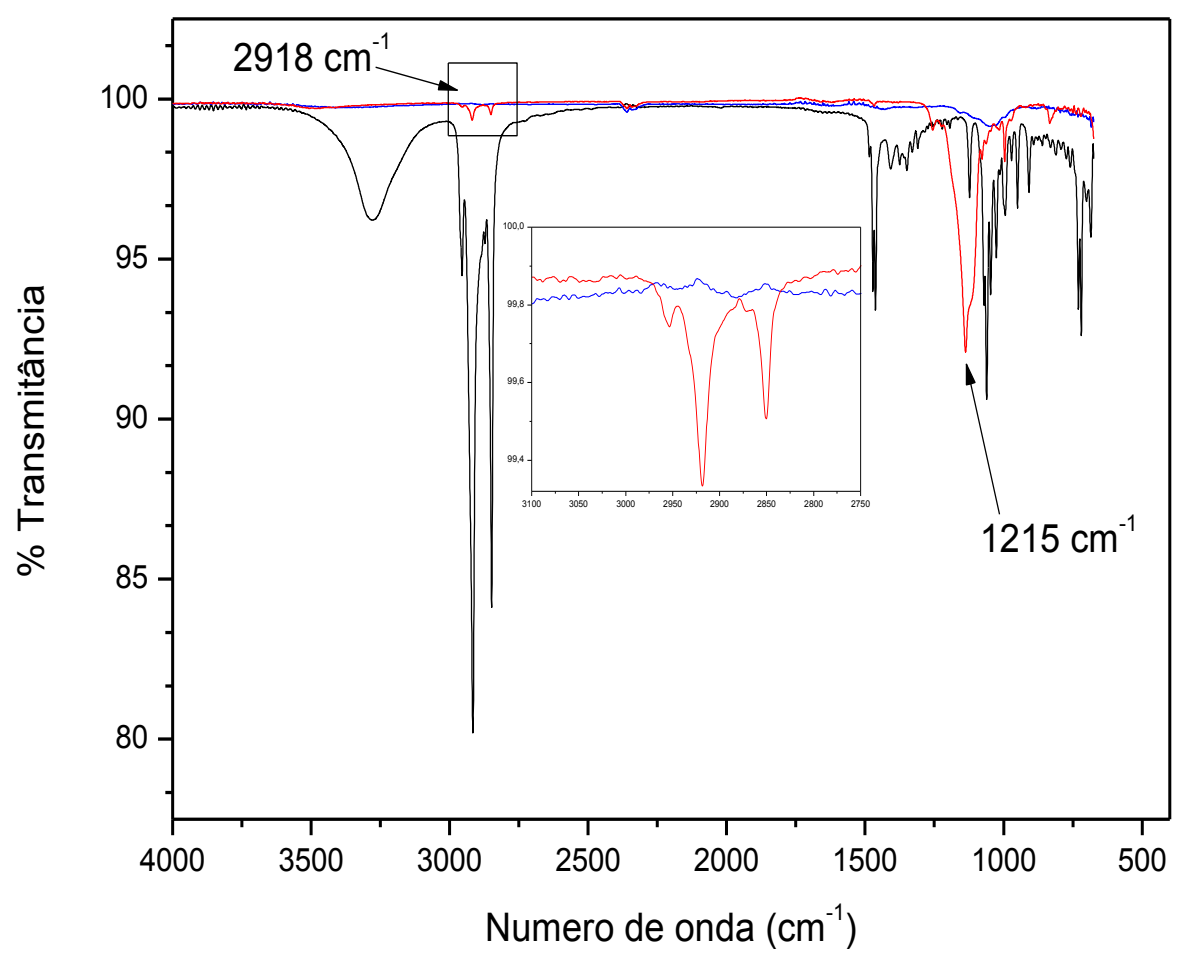

Figura 3: Espectro de infravermelho para celuloses: $(a)(-)$ celulose de sisal bruta, (b) ( $\longrightarrow$ ) celulose de sisal modificada com sulfato, (c) (-) celulose de sisal modificada sem sulfato.

\section{Remoção do íon sulfato}

As Figuras 4 (a) e (b) mostram, respectivamente, o comportamento da solução aquosa do produto obtido na concentração de $1 / 100$ (v/v) após passar no meio filtrante de resina de troca iônica, e a mesma solução antes de passar no referido meio filtrante. Observa-se na Figura 4 (a) a ausência de precipitado e na Figura 4 (b) a presença de precipitação do sulfato de bário, que torna o meio translúcido, o que confirma a eficiência da resina de troca iônica na remoção do íon sulfato.

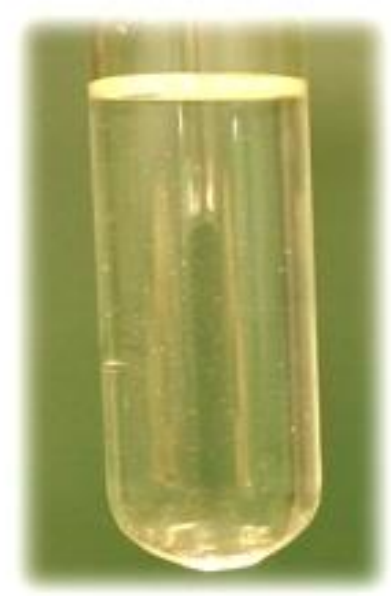

(a)

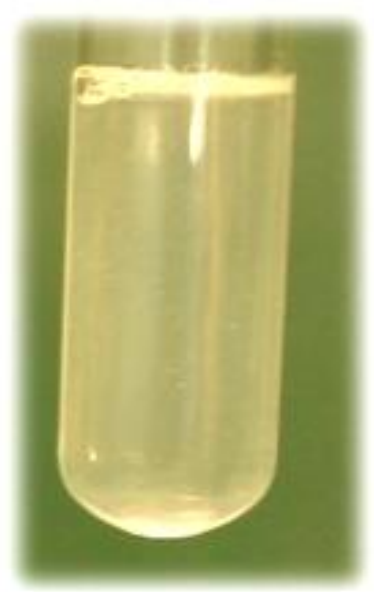

(b)

Figura 4: Solução aquosa de celulose de sisal modificada, (a) após o tratamento de remoção do ion sulfato; $(b)$ antes do tratamento de remocão do íon sulfato. 
A caracterização por FT-ir da celulose de sisal modificada após tratamento de remoção dos íons sulfato é apresentada na Figura 5. Observa-se que mesmo após o processo de remoção do sulfato de sódio, através da utilização da resina, a banda das ligações $\mathrm{C}-\mathrm{H}$ que aparece em torno de $2918 \mathrm{~cm}^{-1}$, presentes no álcool láurico, utilizado como agente modificante, permanece presente na celulose de sisal após sofrer modificação assim como a banda referente à formação da tiocarbonila $(\mathrm{C}=\mathrm{S}) \mathrm{em} 1215 \mathrm{~cm}^{-1}$ proveniente da xantatização. Embora seja verificada a presença de tais bandas, não foi possível através da técnica utilizada, observar a ligação da cadeia alifática do álcool com a celulose xantatiza da conforme mecanismo proposto apresentado na Figura 1.

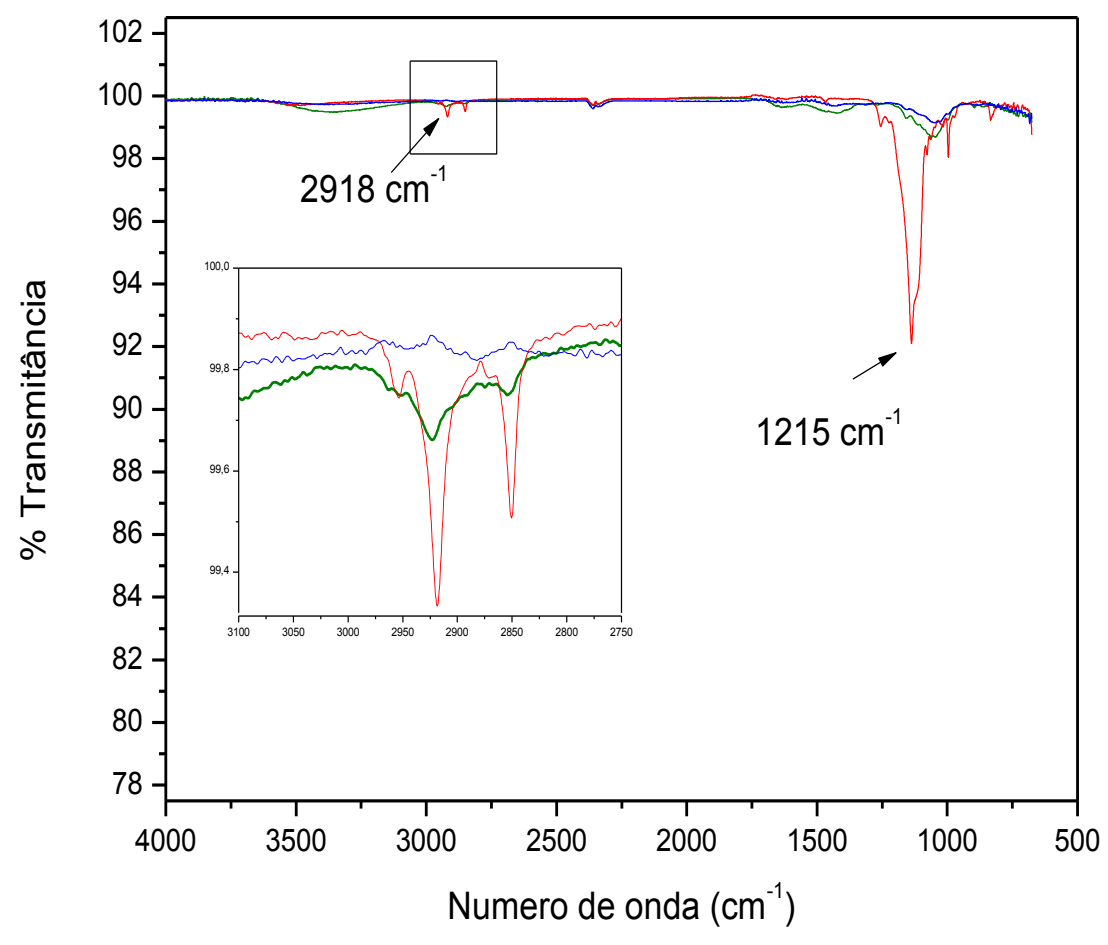

Figura5: Espectro de infravermelho para celuloses: (a) ( — ) celulose de sisal bruta (b) ( celulosede sisal modificada com sulfato (c) (-) celulosede sisal modificada sem sulfato.

\section{Concentração micelar crítica (CMC) da celulose modificada}

A Figura 6 mostra um perfil de condutividade específica versus concentração de celulose modificada. Observa-se que, a partir da concentração zero, à medida que a concentração da celulose modificada é aumentada, a condutividade específica também aumenta linearmente, até atingir o ponto de inflexão em 2,0 g/L que define a CMC. Acima do ponto de inflexão, com a adição da celulose modificada, a condutividade também aumenta linearmente, mas com uma taxa de variação menor do que abaixo da CMC. O aumento linear é forte indicação de que os agregados micelares que se formaram possuem propriedades interfaciais idênticas.

Além da concentração micelar crítica, foi estimado o grau de ionização micelar $(\alpha)$ a partir das razões dos coeficientes angulares das regiões lineares dos gráficos de condutividade específica versus concentração de celulose modificada por meio equação 4 , onde $S_{1}$ e $S_{2}$ referem-se aos coeficientes angulares das regiões lineares abaixo e acima da $\mathrm{cmc}$, respectivamente. Assim sendo, $\alpha$ é igual a $1,74 \mu \mathrm{S} / \mathrm{cm}$.

$$
\alpha=\mathrm{S}_{1} / \mathrm{S}_{2}
$$




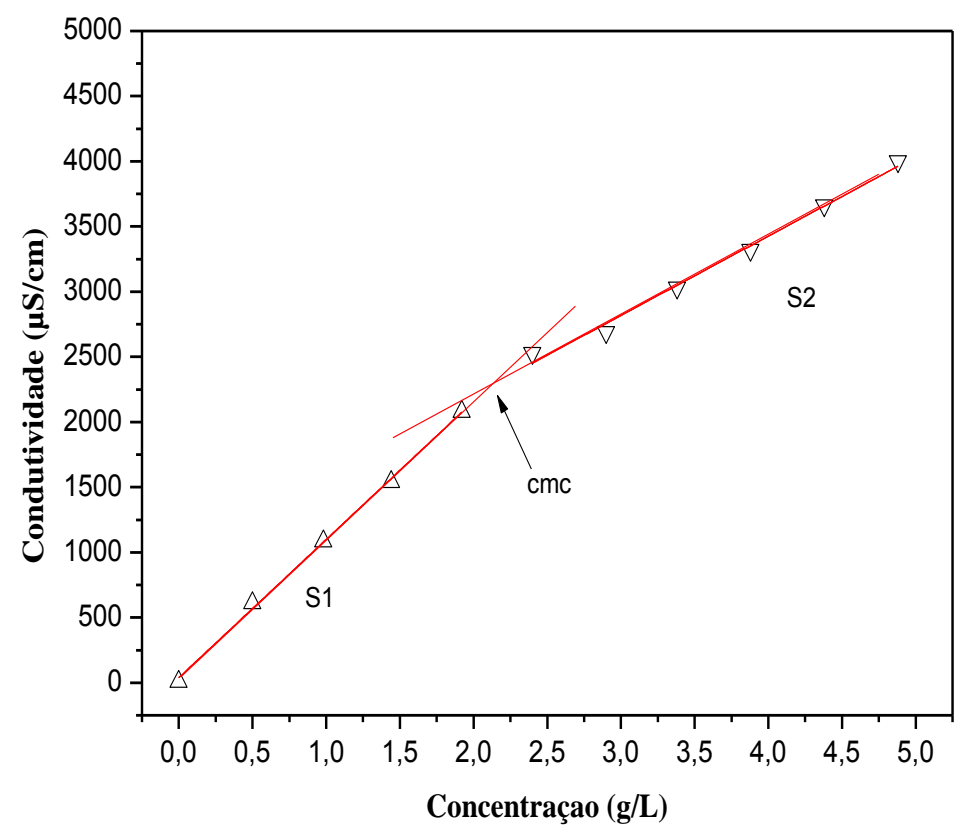

Figura 6: Comportamento da condutividade com a concentração para a celulose de sisal modificada a partir do xantato.

Observou-se também que a celulose de sisal modificada, quando dispersa em água, nas concentrações de 0,5 a 5,0 g/L, conforme Figura 6, não apresentou formação de espuma. O que lhe confere bom potencial para uso no processo de extração e produção de petróleo.

Determinação da temperatura de iniciação da cristalização (TIAC) e da energia de ativação de fluxo $\left(E_{a}\right)$ por reologia

A Tabela 2 mostra os resultados obtidos da TIAC para o óleo parafinico puro e aditivado com a celulose modificada, na ausência e presença de salmoura, respectivamente, obtido a partir de medidas de parâmetros reológicos. Observa-se que a presença do aditivo celulósico não promoveu diminuição na temperatura de iniciação da cristalização das parafinas (TIAC).

Tabela 2: Comportamento da TIAC do óleo em estudo aditivado com celulose modificada com álcool láurico na presença e na ausência de sal.

\begin{tabular}{ccc}
\hline $\begin{array}{c}\text { Concentração do aditivo } \\
(\mathrm{ppm})\end{array}$ & $\begin{array}{c}\text { Na ausência da salmoura } \\
\text { TIAC }\left({ }^{\circ} \mathrm{C}\right)\end{array}$ & $\begin{array}{c}\text { Na presença da salmoura } \\
\text { TIAC }\left({ }^{\circ} \mathrm{C}\right)\end{array}$ \\
\hline 0 & 39 & 39 \\
250 & 39 & 40 \\
500 & 42 & 40 \\
750 & 39 & 38 \\
1000 & 39 & 41 \\
\hline
\end{tabular}

A energia de ativação de fluxo estimada a partir do coeficiente angular da reta de $\ln \mu$ (Pa.s) versus $1 / T(K)$, calculada tomando como referência a TIAC (antes e depois da TIAC), na ausência e presença da salmoura é mostrada na Tabela 3. Observa-se que houve uma diminuição da energia de ativação de fluxo da amostra de petróleo aditivada com a celulose modificada com álcool láurico, na presença da salmoura, após a TIAC, na concentração de 250 ppm, em relação à amostra na ausência de salmoura, que pode ser atribuída a uma maior dispersão dos precipitados, por solvatação. 
Tabela 3:Comportamento da $E_{a}(\mathrm{~kJ} / \mathrm{mol})$ do óleo em estudo aditivado com celulose modificada

\begin{tabular}{c|cc|cc}
\hline \multirow{2}{*}{$\begin{array}{c}\text { Concentração } \\
\text { do aditivo } \\
(\mathrm{ppm})\end{array}$} & \multicolumn{2}{|c|}{$\begin{array}{c}\text { Na ausência de salmoura } \\
\mathrm{E}_{\mathrm{a}}(\mathrm{kJ} / \mathrm{mol})\end{array}$} & \multicolumn{2}{c}{$\begin{array}{c}\text { Na presença de salmoura } \\
\mathrm{E}_{\mathrm{a}}(\mathrm{kJ} / \mathrm{mol})\end{array}$} \\
\cline { 2 - 5 } & Antes da TIAC & Após a TIAC & Antes da TIAC & Após a TIAC \\
\hline 0 & 58 & 71 & 58 & 71 \\
250 & 58 & 74 & 59 & 68 \\
500 & 51 & 68 & 56 & 70 \\
750 & 56 & 76 & 59 & 68 \\
1000 & 58 & 72 & 50 & 68 \\
\hline
\end{tabular}

\section{CONCLUSÃO}

A partir das análises realizadas, concluiu-se que é possível obter substâncias surfactantes com baixo teor de saponificação a partir de modificação de celulose. Para tanto é preciso inicialmente passar a celulose por um processo de xantatização para tornar a sua estrutura suscetível à modificação que atribua ao material característica desejada de surfactante com baixa formação de espuma em virtude da substituição das hidroxilas presentes na celulose pelo agente modificante álcool láurico e pela medida da concentração micelar crítica de $2,0 \mathrm{~g} / \mathrm{L}$ que está na faixa dos valores encontrados por Moraes e Rezende [33].

$\mathrm{O}$ aditivo obtido a partir da celulose de sisal(Agave sisalana) apresentou características desejadas como mostra o parâmetro característico determinado, concentração micelar crítica e baixa saponificação para a utilização de um surfactante facilitador do escoamento de petróleo pesado na presença de sal com composição e concentração do cenário do pré-sal.

\section{AGRADECIMENTOS}

Os autores agradecem ao $\mathrm{CNPq}$ pela concessão da bolsa de mestrado, processo nº133910/2013-0e ao Programa de Pós-Graduação em Engenharia Química da Universidade Federal de Sergipe (PEQ/UFS) pela oportunidade de realização da pesquisa, à SUZANO Papel e Celulose, ao Laboratório de Química Industrial/DEQ-UFS e à Petrobras/UO-SEAL, pelos materiais utilizados neste estudo.

\section{REFERÊNCIAS BIBLIOGRÁFICAS}

1. Wang X, XuS, Tan Y, Du J, and Wang J. Synthesis and characterization of a porous and hydrophobic cellulose-based composite for efficient and fast oil-water separation. Carbohydr. Polym. $2016 \mathrm{Abr}$; 20(140):188-94, doi: 10.1016/j.carbpol.2015.12.028.

2. Alex MT, Rúbia ESM, Eustáquio VRC, Brandão GP, Ricardo JC, Maria TWDC. Extraction induced by emulsion breaking for determination of $\mathrm{Ba}, \mathrm{Ca}$, Mgand $\mathrm{Na}$ in crude oil by inductively coupled plasma optical emission spectrometry. Microchem. J. 2016 Jan;124:338-343, doi.org/10.1016/j.microc.2015.09.014.

3. Klemm D, Heublein B, Fink HP, Bohn A. Cellulose: fascinating biopolymer and sustainable raw material. AngewChem. Int. Ed. Engl. 2005 Mai;44(22):3358-3393, doi: 10.1002/anie.200460587.

4. Wei X,Han Z, Zhang D. Theorical study on the mechanism of the side reaction of 1-butyl-3methylimidazolium cation with D-glucose, Carbohydr. Res. 2013 Jun;374(7):40-44, doi:10.1016/j.carres.2013.03.027.

5. Vershinin VI, Petrov SV. The estimation of total petroleum hydrocarbons content in waste water by IR spectrometry with multivariate calibrations. Talanta. 2016 Fev;1(148):163-169, doi: 10.1016/j.talanta.2015.10.076.

6. Taraneh JB, Rahmatollah G, Hassan A, Alireza D. Effect of wax inhibitors on pour point and rheological properties of Iranian waxy crude oil. Fuel Process. Technol. 2008 Fev;89(10):973-977, doi:10.1016/j.fuproc.2008.03.013. 
7. Soni HP, Kiranbala, AGRAWAL K.S.; NAGAR A.; BHARAMBE D.P. Designing maleic anhydride$\alpha$-olifincopolymeric combs as wax crystal growth nucleators. Fuel Process. Technol. 2010 Set;91(9):997-1004, doi: 10.1016/j.fuproc.2010.02.019.

8. Zhang R, Yang L,Tu R, Huo J, Wang J, Zhou J, Chen D. Emulsion phase inversion from oil-in-water (1) to water-in-oil tooil-in-water (2) induced by in situ surface activation of $\mathrm{CaCO}_{3}$ nanoparticles via adsorption of sodium stearate. Colloids and Surfaces A: Physicochem. Eng. Aspects. 2015 Jul; 477(20):55-62, doi: 10.1016/j.colsurfa.2015.03.043.

9. Dantas NAA, Gomes EAS, Barros NEL, Dantas TNC, Moura CPAM. Determination of wax appearance temperature (WAT) in paraffin/solvent systems by photoelectric signal and viscosimetry. Braz. J.Petroleum Gas. 2010;3(4):149-157, doi: 10.5419/bjpg.v3i4.109.

10. Schaefer J, Lee G. Arrhenius activation energy of damage to catalase during spray-drying. Int. J. of Pharm.2015 Jul;489(1-2):124-130, doi:10.1016/j.ijpharm.2015.04.078.

11. Coto B, Martos C, Pena JL, Espada JJ, Robustillo MD.A new method for the determination of wax precipitation from non-diluted crude oils by fractional precipitation. Fuel. 2008 Ago;87(10-11):20902094,doi:10.1016/j.fuel.2007.12.012.

12. Liu CF, Zhang AP, Li WY, Yue FX, Sun RC. Succinolation of cellulose catalyzed with iodine in ionic liquid. Ind. Crops and Prod. 2010 Fev;31(2):363-369, doi: 10.1016/j.indcrop.2009.12.002.

13. Liu CF, Zhang AP, Li WY, Yue FX, Sun RC. Homogeneous modification of cellulose in ionic liquid with succinic anhydride using $n$-bromosuccinimide as a catalyst. J. Agric. Food Chem. 2009;57:18141820, doi: 10.1021/jf803097k.

14. Li S, Zhang S, Wang X. Fabrication of superhydrophobic cellulose-based materials through a solutionimmersion process. Langmuir. 2008 Abr;24(10):5585-5590,doi: 10.1021/la800157t.

15. Jin $\mathrm{H}$, Zha $\mathrm{C}$, GU L. Direct dissolution of cellulose in $\mathrm{NaOH} /$ thiouréia/uréia aqueous solution. Carbohidr. Res., 2007 May;342(6):851-858, doi: 10.1016/j.carres.2006.12.023.

16. Motokawa T, Makino M, Enomoto-Rogers Y, Kawaguchi T, Ohura T, Iwata T, Sakaguchi M. Novel method of the surface modification of the microcrystalline cellulose powder with poly(isobutyl vinyl ether) using mechanochemical polymerization. Adv. Powder Technol. 2015 Set;26(5):1383-1390,doi: 10.1016/j.apt.2015.07.012.

17. Gallego R, Arteaga JF, Valencia C, Franco JM. Chemical modification of methyl cellulose with hdmi to modulate the thickening properties in castor oil. Cellulose. 2013 Fev; 20(1):495-507, doi: 10.1007/s10570-012-9803-4.

18. Li WY, Jin XB, Liu CF, Sun RC, Zhang AP, Kennedy JF. Homogeneous modification of cellulose with succinic anhydride in ionic liquid using 4- dimethylaminopyridine as a catalyst. Carbohydr. Polym. 2009 Out;78(3):389-395, doi:10.1016/j.carbpol.2009.04.028.

19. Xiao D, Yang F, Han H, Liu Z, Zhang S, LI G. Preparation of oligosaccharide surfactant based on cotton cellulose and application in hydrophilic modification of docetaxel. Colloids and Interface Sci. Commun. 2015 Jan;4:10-13, doi:10.1016/j.colcom.2015.04.002.

20. Song Y, Jinping Z, Zhang L, Wu X. Homogenous modification of cellulose with acrylamide in $\mathrm{NaOH} /$ Uréia aqueous solutions. Carbohydr. Polym. 2008 Jul;73(1):18-25, doi:10.1016/j.carbpol.2007.10.018.

21. Al-Yaari M, Hussein IA, Al-Sarkhi A, Abbad M, Chang F. Effect of water salinity on surfactantstabilized water-oil emulsions flow characteristics. Exp. Therm.Fluid Sci. 2015 Fev;64:5461,doi:10.1016/j.expthermflusci.2015.02.001.

22. Wei Y, Cheng F, Hou G, Sun S. Amphiphilic cellulose: Surface activity and aqueous self-assembly into nano-sized polymeric micelles. React. \&Funct. Polym. 2008 Mai; 68(5):981-989, doi:10.1016/j.reactfunctpolym.2008.02.004.

23. Deng W, Zhang Q, Wang Y. Catalytic transformations of cellulose and cellulose-derived carbohydrates into organic acids. Catal. Today. 2014 Out;234(1):31-41, doi:10.1016/j.cattod.2013.12.041.

24. Corti GS. Estudo da capacidade de complexação de íons $\mathrm{Cu}^{2+}$ em solução aquosa usando celulose modificada com anidrido succínico e com poliaminas. Polim.:Cienc. Tecnol. 2004 Out;14(5):313-317, doi: 10.1590/S0104-14282004000500007.

25. Jia X, Chen Y, Shi C, Ye Y, Wang P, Zeng X, Wu T. Preparation and Characterization of Cellulose Regenerated from Phosphoric Acid. J. Agric. Food Chem. 2013 Nov;61(50):12405-12414, doi: $10.1021 / \mathrm{jf} 4042358$.

26. Okoro IA, Ejike EN. Chemical modification cellulose pulp as crude oil absorvent.Res. J. App. Sci. 2007;2(1):75-76, doi: rjasci.2007.75.76.

27. Hirano S, Usuta A, and Midorikawa T. Novel fibers of iv-acylchitosan and its cellulose composite prepared by spinning their aqueous xanthate solutions. Carbohydr. Polym. 1997 Mai;33(1):1-14, doi: 10.1016/S0144-8617(97)00039-8.

28. Pessoa AJr, Kilikian BV. Purificção de produtos biotecnológicos. 1 edição. 2005 Barueri-SP. 404p. 
29. Zanette D, Frescura V. Ideal Mixing of Polymer-Surfactant Complexes of Polyethylene Oxide and Sodium Dodecyl Sulfate Plus Sodium Dodecanoate. Colloids and Surfaces. 1999 Mai;213(2):379-385, doi: 10.1006/jcis.1999.6115.

30. SchröderJ, Kleinhans A, Serfert Y, Drusch S, Schuchmann HP,Gaukel K. Viscosity ratio: A key factor for control of oil drop size distributionin effervescent atomization of oil-in-water emulsions. J. FoodEng. 2012 Jun;111(2):265-271, doi:10.1016/j.jfoodeng.2012.02.023.

31. ASA-Applied Science Associates South American.Modelagem do Descarte de Efluentes a partir dos Projetos Piloto de Guará e Desenvolvimento da Produção de Iracema, Bacia de Santos. Rel.Técn., 2011.

32. Silverstein RM, Webster FX, Kiemle DJ. Identificação espectrométrica de compostos orgânicos. Rio de Janeiro: LTC, 2007. Vol. Único. 490p.

33. Moraes SL, Rezende MOO. Determinação da concentração micelar crítica de ácidos húmicos por medidas de condutividade e espectroscopia. Quim Nova. 2004 Jul;27(5):701-705. 